\title{
Establishing HZ43 A, Sirius B, and RX J185635-3754 as soft X-ray standards: a cross-calibration between the Chandra LETG+HRC-S, the EUVE spectrometer, and the ROSAT PSPC ${ }^{\star} \star \star \star$
}

\author{
K. Beuermann ${ }^{1}$, V. Burwitz ${ }^{2}$, and T. Rauch ${ }^{3}$ \\ ${ }^{1}$ Institut für Astrophysik, Friedrich-Hund-Platz 1, 37077 Göttingen, Germany \\ e-mail: beuermann@astro.physik.uni-goettingen.de \\ 2 MPI für Extraterrestrische Physik, 85748 Garching, Germany \\ e-mail: burwitz@mpe.mpg.de \\ 3 Institut für Astronomie und Astrophysik, Sand 1, 72076 Tübingen, Germany \\ e-mail: rauch@astro.uni-tuebingen.de
}

Received 22 April 2006 / Accepted 29 June 2006

\section{ABSTRACT}

\begin{abstract}
Context. The absolute calibration of space-borne instruments in the soft X-ray regime rests strongly on model spectra of hot white dwarfs.

Aims. We analyze the Chandra LETG+HRC observations of the white dwarfs HZ43 A and Sirius B and of the neutron star RX J185635-3754 with the aim of resolving current uncertainties in the soft X-ray spectral fluxes and photospheric parameters of the three stars. We apply the derived photon spectra to a cross-calibration of the LETG+HRC-S with the short-wavelength EUVE spectrometer and the ROSAT PSPC.

Methods. We tie HZ43 A to the flux of RX J1856 in the 44-48 A range and perform a simultaneous least squares fit to the LETG+HRC spectra of the three stars. This allows us to determine an internally consistent set of spectral energy distributions and an empirically derived wavelength-dependent correction to the LETG+HRC-S effective area. We employ NLTE model atmospheres calculated with TMAP for the white dwarfs and a two-blackbody model for RX J1856, tied to the respective optical fluxes.

Results. The two-blackbody model for RXJ1856 features a hot spot on a cooler star and yields $k T_{\text {spot }}=62.8 \pm 0.4 \mathrm{eV}$ and $k T_{\text {star }}=32.3 \pm 0.7 \mathrm{eV}$ with a stellar angular radius as seen from infinity of $0.1371 \pm 0.0010 \mathrm{~km} \mathrm{pc}^{-1}$. For HZ43 A, our fit yields $T_{\text {eff }}=51126 \pm 660 \mathrm{~K}$ and $\log g=7.90 \pm 0.080(\mathrm{cgs})$ with anti-correlated errors $(1-\sigma)$ which include not only the statistical but also the systematic uncertainties of the fit. HZ43AB displays a previously detected bremsstrahlung component with a temperature $k T \simeq 0.6 \mathrm{keV}$. For Sirius B, we find $T_{\text {eff }}=24923 \pm 115 \mathrm{~K}$ for fixed $\log g=8.6$. The calibration of the short-wavelength $E U V E$ spectrometer differs from that of the LETG+HRC-S by $15 \pm 7 \%$. The ROSAT PSPC is found to be correctly calibrated within a few percent and reports of a major miscalibration are unfounded.

Conclusions. We have obtained improved parameters for RXJ185635-3754, HZ43 A, and Sirius B which fit the observations from the optical to the soft X-ray regime. Our approach allows us to quote their absolute spectral fluxes at selected wavelengths which may aid the calibration of other space-borne instruments.
\end{abstract}

Key words. methods: data analysis - white dwarfs - stars: neutron - stars: fundamental parameters

\section{Introduction}

Hot white dwarfs with pure hydrogen atmospheres are well established standard stars in the optical and ultraviolet (e.g. Bohlin et al. 2001) and have played a decisive role in the calibration of satellite instruments in all spectral regimes from the optical to the extreme ultraviolet and soft X-rays. The FOS and STIS on the Hubble Space Telescope (Bohlin 2000), the Extreme Ultraviolet Explorer (EUVE) (Boyd et al. 1994), and the Low Energy Transmission Grating Spectrometer on board Chandra (LETG+HRC-S) with the High Resolution Camera as detector (Brinkman et al. 2000; Pease et al. 2000) are prominent

* Based on observations collected with the Chandra Observatory, the Hubble Space Telescope, the EUVE satellite, and ROSAT.

$\star \star$ Tables A. 1 to A.6 are only available in electronic form at the CDS via anonymous ftp to

cdsarc.u-strasbg.fr $(130.79 .128 .5)$ or via

http://cdsweb.u-strasbg.fr/cgi-bin/qcat?J/A+A/458/541 examples. The latter two instruments were calibrated on the ground, but their calibration was partly relinquished in favor of an in-flight calibration using hot white dwarfs. The Position Sensitive Proportional Counter (PSPC) on board the Röntgen Satellite (ROSAT), on the other hand, was accurately calibrated on the ground (Trümper 1982; Pfeffermann et al. 1987) and not recalibrated in flight. Based on observed PSPC spectra of white dwarfs, however, Napiwotzki et al. (1993); Jordan et al. (1994); Wolff et al. (1995, 1996) claimed the PCPC calibration at soft $\mathrm{X}$-ray energies to be incorrect by a factor of about two. Given this important role of white dwarfs it is deplorable that their soft $\mathrm{X}$-ray fluxes are much less well documented than their optical and ultraviolet fluxes. Given, furthermore, the large body of scientific data of all types of objects potentially affected, the resolution of such open calibration issues is of general interest.

Our initial aim was to investigate the PSPC calibration which requires, however, to take recourse to spectrally higher resolved data as provided by the EUVE or the Chandra LETG+HRC 
spectrometers. Since the effective area of the latter is itself uncertain by as much as $25 \%$ at intermediate wavelengths around $60 \AA$ (e.g. Pease et al. 2003), we found it necessary to perform a more detailed cross-calibration of the three instruments. As a result, we are able to determine accurate soft X-ray fluxes of the hot white dwarfs HZ43 A and Sirius B and of the neutron star RX J185635-3754 (henceforth RX J1856) and thereby establish them as standards in the soft X-ray regime ${ }^{1}$. Although one of the two white dwarfs (HZ43 A) is a well known standard for wavelengths $\lambda>912 \AA$ (Bohlin et al. 2001) and the optical flux of the other (Sirius B) is reasonably well measured (Barstow et al. 2005), the extrapolation of the optically adjusted model spectra into the soft X-ray regime suffers from the wagging-tail problem. We solve this problem by tying the soft X-ray tail of the spectrum of HZ43 A to that of RX J1856. This adds a second fix to the spectrum of HZ43 A besides that in the optical and effectively constrains its spectral shape.

The organization of the paper is as follows. In Sect. 2, we describe the analysis of the LETGS and PSPC data and list the EUVE archive data used. Section 3 is devoted to the description of our theoretical white dwarf spectra and summarizes the available information on $T_{\text {eff }}$ and $\log g$ of HZ43 A. Our principal results are provided in Sect. 4. It starts with information on the optical fluxes to which our model spectra are normalized (Sect. 4.1). In Sect. 4.2, we derive an accurate spectrum of RX J1856 that serves as the short-wavelength fix for HZ43 A. In this context, we discuss interstellar absorption near the carbon K-edge at $43.7 \AA$ to some detail. The essence of the paper is then presented in Sect. 4.4, the simultaneous fit of RX J1856, HZ43 A, and Sirius B that results in an internally consistent set of photon spectra and simultaneously in the wavelengthdependent correction functions to the LETG+HRC-S effective areas at wavelengths $\lambda \geq 44 \AA$. Here, we discuss also the systematic errors of our approach. The stellar parameters implied by our fit are presented and discussed in Sect. 4.5. Section 4.6 compares our LETGS calibration to that of the EUVE short wavelength spectrometer and Sect. 4.7 finally demonstrates the accurate calibration of the ROSAT PSPC. The conclusions are summarized in Sect. 5.

\section{Observations and data analysis}

We have extracted all observations of HZ43 A, Sirius B, and RX J1856 taken with the Chandra LETG+HRC-S, the EUVE spectrometer, and the ROSAT PSPC from the respective archives. Table 1 contains a log of the observations.

\subsection{Chandra LETG+HRC spectra}

The spectra were extracted from the reprocessed level 2.0 event files. The corresponding total exposure times are listed in Table 1. The extraction region recommended in the Chandra Proposers' Observation Guide was used and the background determined from wide regions below and above the source extraction area. Further details are given by Burwitz et al. (2003). Since the background is variable, we investigated the influence of rigorously discarding intervals of high background on the resulting count rate spectra. Our tentative elimination scheme (in short referred to as level 3) reduces the exposure times of RX J1856, HZ43 A, and Sirius B to $66 \%, 84 \%$, and $61 \%$ of the level 2.0

\footnotetext{
1 We consider neither quasars and BL Lacs because of their inherent variability nor supernova remnants because of their extended nature.
}

Table 1. Journal of observations extracted from the Chandra, EUVE, and ROSAT archives for the present analysis. Only the total exposure times are given, except for the ROSAT PSPC where the experimental setups differ and the individual exposure times are given.

\begin{tabular}{|c|c|c|c|}
\hline Object & Obs. Identif. & Year & Exp. (ks) \\
\hline \multicolumn{4}{|c|}{ a) Chandra LETG+HRC-Spectrometer } \\
\hline HZ43 A & $\begin{array}{l}00059,01011,01012, \\
02584,02585,03676, \\
\text { 03677, 05042, 05044, } \\
\text { 05957, 05959, 06473 }\end{array}$ & $\begin{array}{l}1999,2001 \\
2002 \\
2003,2004 \\
2005,2006\end{array}$ & 254.8 \\
\hline Sirius B & $01421,01452,01459$ & 1999,2000 & 64.1 \\
\hline RX J1856 & $\begin{array}{l}\text { 00113, 03380, 03381, } \\
\text { 03382, } 03399\end{array}$ & 2000,2001 & 501.9 \\
\hline \multicolumn{4}{|c|}{ b) EUVE-Spectrometer } \\
\hline HZ43 A & $\begin{array}{l}1904 \mathrm{~N}, 1620 \mathrm{~N} \\
1049 \mathrm{~N}, 0030 \mathrm{~N}\end{array}$ & $\begin{array}{l}1994,1995 \\
1996,1997\end{array}$ & 130.0 \\
\hline Sirius B & $0147 \mathrm{~N}, 0039 \mathrm{~N}$ & 1993,1996 & 246.0 \\
\hline \multicolumn{4}{|c|}{ c) $R O S A T P S P C$} \\
\hline \multirow[t]{2}{*}{ HZ43 A } & $\begin{array}{l}\text { rp100308 } \\
\text { rf200418 }\end{array}$ & $\begin{array}{l}1990 \\
1991\end{array}$ & $\begin{array}{l}21.5 \\
21.6\end{array}$ \\
\hline & rp141916/17 & 1992 & 6.9 \\
\hline Sirius B & rf200422 & 1991 & 3.2 \\
\hline RX J1856 & rp200497 & 1992 & 6.3 \\
\hline
\end{tabular}

accepted times, respectively, and is more restrictive than the condition which Drake et al. (2002) imposed on the accepted telemetry rate. Nevertheless, the wavelength-integrated count rates increase by only $0.8 \%, 0.1 \%$, and $6.8 \%$, respectively. These values measure the additional dead-time resulting from the time intervals of enhanced background. We opt to accept the level 2.0 spectra with the small dead-time corrections applied to them as a multiplicative factor. Within their statistical errors, the level 3 and the dead-time corrected level 2.0 spectra are indistinguishable from one another.

The long series of spectra taken of HZ43 A between 1999 and 2006 lends itself to an analysis of the long-term behavior of the HRC sensitivity. The zeroth order count rate of HZ43 A decreases slowly with time at a rate of $0.98 \pm 0.15 \%$ per year or with a time scale of about 100 years. This decrease seems to depend on wavelength, with a low of the decay rate of $0.5 \%$ per year near $110 \AA$, rates of $0.9 \pm 0.2 \%$ for $70-80 \AA$, and a more uncertain $2.4 \pm 0.8 \%$ for $44-49 \AA$ (Fig. 1). For Sirius B and RX J1856, the time bases are much more restricted as indicated by the tick marks in Fig. 1. The decay rates of their zeroth order count rates are $\sim 0.7 \%$ and close to zero, respectively. The spatial and temporal gain changes of the HRC-S are a known effect and a study for times before JD 2452900 has been presented by Pease \& Drake (2003). We do not attempt to correct for these changes, but rather chose a pragmatic approach. We place a simultaneous spectral fit on a secure basis by selecting a subset of the HZ43 A exposures with an epoch nearly equal to that of the RX J1856 observations, i.e., the exposure-weighted mean Julian day JD 2452130 . We use the HZ43 A exposures 00059 through 03677 which comprise $157239 \mathrm{~s}$ of exposure with an epoch different from that of RX J1856 by only 38 days. A similar selection is not possible for Sirius B which is centered 508 days earlier. We do not correct for the possible $1 \%$ effect.

We start using the first-order effective areas of the LETG+HRC-S in the positive and negative dispersion directions as recommended in the November 2004 release of the 


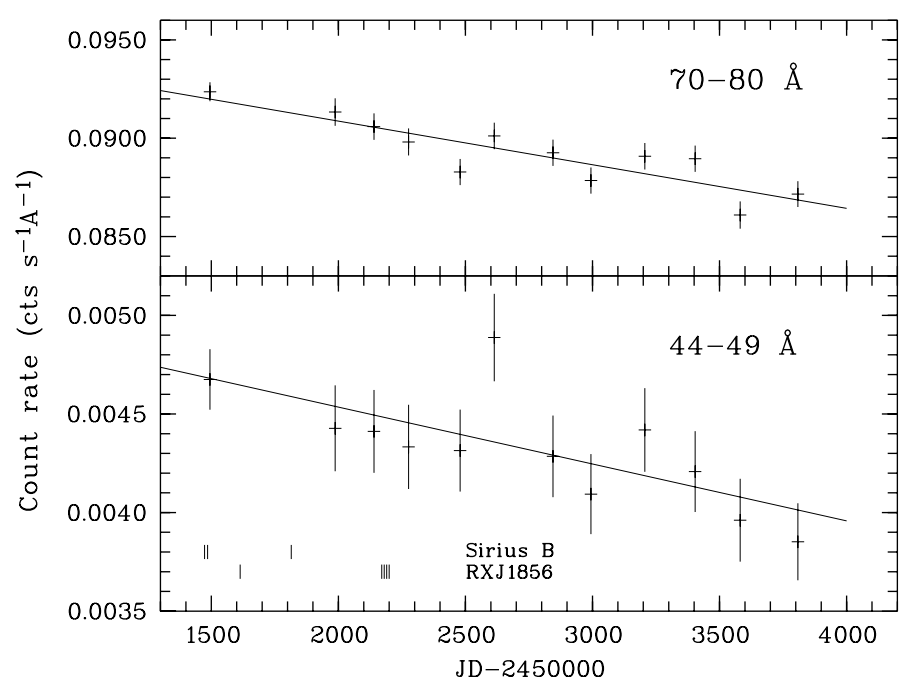

Fig. 1. Long-term variation of the integrated level 2.0 LETG+HRC count rates of HZ43 A for two spectral intervals. The two sets of tick marks indicate the times when the LETG+HRC spectra of Sirius B and RX J1856 were taken.

Chandra X-ray Center ${ }^{2}$ and refer to them as the nominal areas $A^{0}$. Throughout this paper, we use the efficiencies $\varepsilon_{k}$ of orders $k=2$ to 6 relative to the first order as given in the same release. The areas $A^{0}$ are reliable to better than $15 \%$ shortwards of the carbon K-edge at $\lambda=43.7 \AA$, but may need corrections possibly as large as $25 \%$ in the $60-80 \AA$ regime (Pease et al. 2003). We distinguish, therefore, $A^{0}$ from $A=\alpha A^{0}$, where $\alpha(\lambda)$ is a wavelength dependent adjustment factor which equals unity shortwards of the carbon K-edge and is allowed to deviate from unity longwards of it. Our correction function $\alpha$ derived below is strictly valid only for the epoch JD 2452130 and may differ for other epochs during the lifetime of the HRC at the percent level.

The calibration of the LETG+HRC-S at long wavelengths is based, in part, on Sirius B and HZ43 A (Brinkman et al. 2000; Pease et al. 2000), with a certain preference given to Sirius B because of its better defined $\log g$ (Barstow et al. 2005; Holberg et al. 1998). The drawback is the lack of a useful signal from Sirius B at $\lambda<60 \AA$, while HZ43 A can be detected at least down to $\lambda=44 \AA$. Since we wish to connect the spectrum of our white dwarf calibrator to the harder spectrum of RX J1856, we give preference to $\mathrm{HZ} 43 \mathrm{~A}$. This has the added advantage that RX J1856 and HZ43 A are both observed on-axis while the Sirius pointings are slightly off axis. The latter may affect the simultaneous fit if the HRC efficiency differs spatially (in addition to temporally) for a given wavelength. We neglect this possible effect.

We bin the LETG+HRC spectra to $0.5 \AA$ in wavelength which is an adequate compromise between spectral resolution and statistical significance of individual data points in the less well exposed wavelength regions. The detector gaps for the observations of RX J1856 and HZ43 A are conservatively defined as $49-58 \AA$ and $58-69 \AA$ for the negative and positive dispersion directions, respectively. For Sirius $B$, the gaps overlap and statistically significant data of exist only for $\lambda \geq 61 \AA$.

\footnotetext{
${ }^{2}$ http://cxc.harvard.edu/cal/Letg $\rightarrow$ LETG/HRC-S Effective Area (updated November 2004).
}

\subsection{EUVE spectra}

We obtained six night time EUVE spectra of HZ43 A and two of Sirius B. The archived photon spectra were used and we refrained from a re-extraction of the data. The archived spectra are based on corrected effective areas as described by Boyd et al. (1994). Two spectra of HZ43 A (9302180059, 9403252311) were discarded because of excessive noise and low flux, respectively. The remaining four are listed in Table 1 . They differ in their general flux levels by $\pm 5 \%$, but no systematic time variation is seen. We did not use the very faint and ill-determined EUVE spectrum of RX J1856 discussed by Pons et al. (2002).

\subsection{ROSAT PSPC spectra}

All ROSAT spectra of HZ43 A, Sirius B, and RX J1856 taken with the Position Sensitive Proportional Counter (PSPC) were extracted from the ROSAT archive and re-analyzed. Since the three sources are very bright, we used a generous extraction radius of 5 arcmin radius which was only reduced for the determination of the high energy bremsstrahlung tail of HZ43 A (O'Dwyer et al. 2003). The background was taken, if possible, from a surrounding ring free of additional faint sources. We list in Table 1 the subset of observations discussed in detail here, one each from the 1990 Program Verification (PV) phase, the 1991 Announcement of Opportunity 1 (AO-1), and the 1992 AO-2. The PV observations were taken with PSPC-C and the AO-1 observations with PSPC-B before the reduction of the high voltage on October 11,1991 . The detector response matrix for both data sets is DRMPSPC-AO1 (Briel et al. 1995). For the AO-2 observations after the voltage change, the appropriate matrix is DRMPSPC. In order to allow a straightforward comparison with the previous analyses of Wolff et al. (1996, and references therein), we do not apply any adjustments to the matrices and refrain from corrections for the known (small) temporal and spatial gain shifts of the PSPC (Prieto et al. 1996; Snowden et al. 2001). For Sirius B and RX J1856 only one PSPC observation each was performed, of which the former is in AO- 1 and the latter in AO-2. The observations with identification numbers starting with rp were taken in the open configuration, the ones starting with rf include the boron filter which reduces the count rate for the very soft white dwarfs by about a factor of eight as a result of the added absorption and the narrower width of the response in photon energy.

All integrated PSPC count rates quoted in Sect. 4.7 below refer to energy channels 11-60 (of 256). We note already here that our count rates are lower than those presented by Wolff et al. (1996) who seem to have included channels below the recommended lower limit of channel 11.

\section{Model atmospheres and the case of HZ43}

In this section, we describe the non-LTE model atmospheres used in our analysis and summarize previous results on the photospheric parameters of HZ43.

\subsection{NLTE models for pure hydrogen atmospheres}

We employ TMAP, the Tübingen NLTE Model Atmosphere Package (Werner et al. 2003; Rauch \& Deetjen 2003), in its most recent version for the calculation of plane-parallel, static, pure-hydrogen models. Hydrogen is represented by a model atom with the lowest levels up to principal quantum number $n=14$ treated in NLTE. The higher levels are dissoluted rapidly 


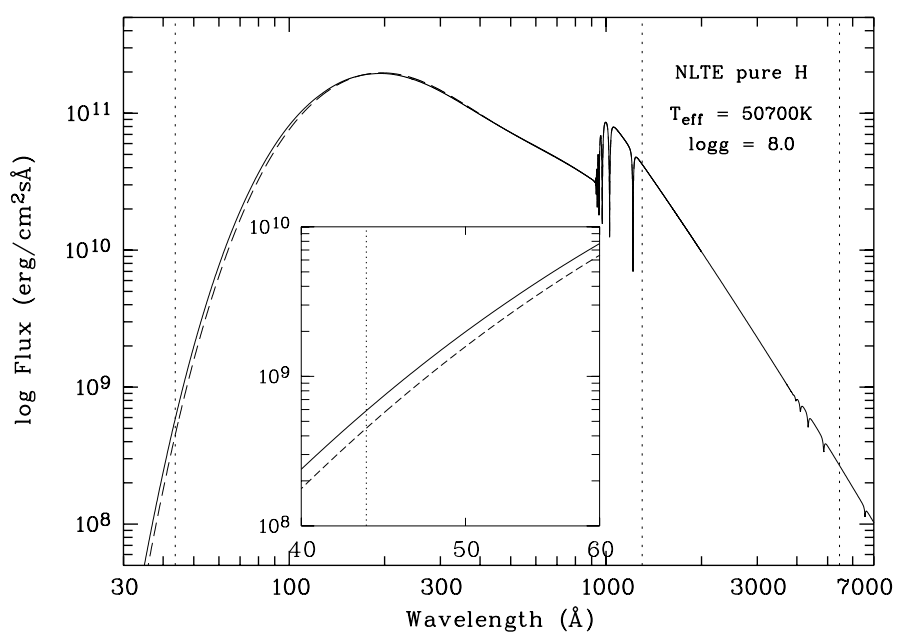

Fig. 2. NLTE pure hydrogen spectra for $T_{\text {eff }}=50700 \mathrm{~K}$ and $\log g=8.0$, calculated with TMAP using two versions of the bound-free and freefree absorption coefficients, Seaton's approximation (dashed curve) and the full Karszas \& Latter (1961) description (solid curve). Both models yield practically identical results at optical/ultraviolet wavelengths, but differ by a factor of 1.33 at $44 \AA$ (vertical dotted line). The insert shows an expanded view. Two further dotted lines mark $\lambda=1300 \AA$ and $\lambda=$ $5450 \AA$ (see text).

towards the inner photosphere at the high $g$ considered here, e.g., to less than $9 \%$ occupation probability at a column density of only $10^{-5} \mathrm{~g} \mathrm{~cm}^{-2}$ for $n=14$ and the $T_{\text {eff }}=27900 \mathrm{~K}$ and $\log g=8.6$ of Sirius B (Hummer \& Mihalas 1988). Hence, this number of NLTE levels is sufficient. Two additional levels are treated in LTE. The NLTE levels are fully coupled by radiative line transitions. An approximate formula for the Stark broadening (Unsöld 1968) is employed for the calculation of their absorption coefficients. Collisional transitions are considered between all levels. The atmospheres are calculated within optical depths of $\log \tau=-8 \ldots+4$. In order to model the flux at the Lyman threshold precisely within 900-1000 A, a narrow frequency grid was used with a spacing equivalent to $0.1 \AA$. For the synthetic spectra, we use the line-broadening tables of Lemke (1997).

The short-wavelength continuum benefits from the use of $f f$-cross sections from Sutherland $(1998)^{3}$ which replace the older fit formula from Mihalas (1967). More importantly, the continuum flux depends on the treatment of the $b f$-transitions. Using the full formalism of Karzas \& Latter (1961) raises the flux at $44 \AA$ by $33 \%$ compared with spectra calculated with the older Seaton formula and Gaunt factor (see Fig. 2). We find very good agreement of our results with the short-wavelength continuum of Barstow et al. (2003). Given the sensitivity of the soft $\mathrm{X}$-ray fluxes to details of the calculation, we caution against the indiscriminate comparison of different versions of model spectra used over the decades in the calibration of various satellite instruments sensitive in the soft X-ray regime.

Another aspect suggested to be important at soft X-ray energies is the suppression of the flux by Compton rather than Thomson scattering. Although Madej (1998), claimed a sizeable effect, Suleimanov et al. (2006) show that it is only at the $1 \%$ level near the carbon K-edge in HZ43 A and Sirius B. Since

\footnotetext{
${ }^{3}$ http://astro.uni-tuebingen. de/ rauch/TMAP/UserGuide/ UserGuide.html
}

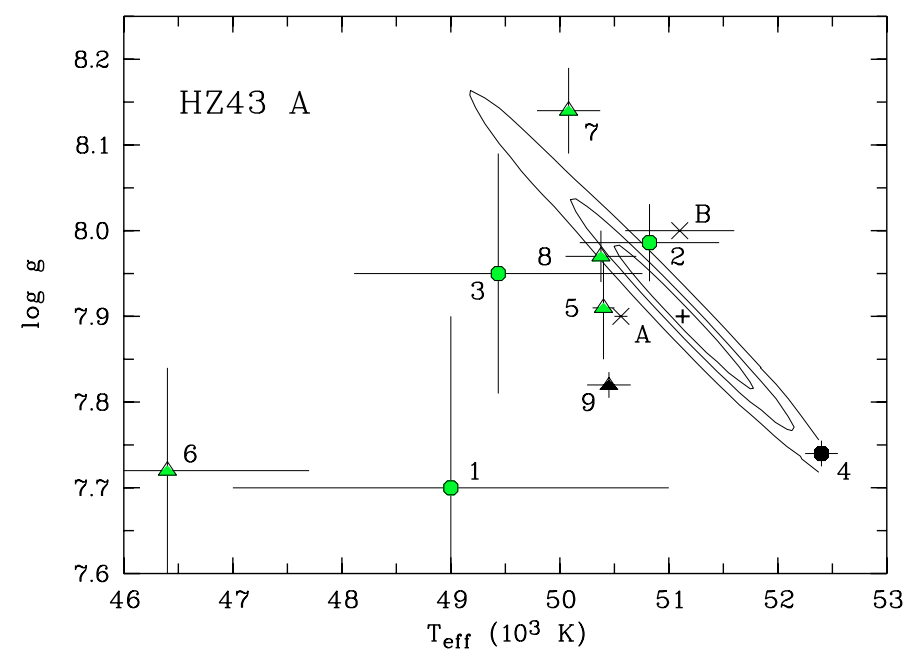

Fig. 3. Summary of $T_{\text {eff }}-\log g$ determinations for HZ43 A from fits to the Balmer lines (circles), the Lyman lines (triangles), and the EUVE continuum (lying crosses): $1=$ Napiwotzki et al. (1993); $2=$ Finley et al. (1997); $3=$ Barstow et al. (2003); $4=$ this work (STIS Balmer lines); $5=$ Dupuis et al. (1998) (ORFEUS); $6=$ Barstow et al. (2001) $($ HUT $) ; 7=$ Barstow et al. (2001) $($ ORFEUS); $8=$ Barstow et al. (2003) (FUSE); $9=$ this work (FUSE); A = Barstow et al. (1995) (EUVE); and $\mathrm{B}=$ Vennes \& Dupuis (2002) (EUVE). The error ellipses indicate the results of the present fits to the Chandra soft X-ray continuum and refer to the $68 \%, 90 \%$ and $95 \%$ confidence levels.

the Compton effect deforms the model spectrum, we investigate the effect quantitatively below.

\subsection{The case of $H Z 43 A$}

Figure 3 summarizes previous measurements of $T_{\text {eff }}$ and $\log g$ of HZ43 A supplemented by our present results. The individual parameter combinations scatter over $6000 \mathrm{~K}$ and $0.4 \mathrm{dex}$, respectively, with no preference for any individual method to prefer a particular region in parameter space. Systematic differences in the deduced parameters from Lyman and Balmer lines were extensively studied by Barstow and collaborators, who show that they are still small near $50000 \mathrm{~K}$ (Good et al. 2004). The data points in Fig. 3 cluster around $T_{\text {eff }}=50500 \mathrm{~K}$ and $\log g=7.95$, but it is not certain that such a poll bears physical significance. The cause for the partly disparate data points is likely systematic in nature and may have its origin in observational problems, e.g., the presence of stray light in the line centers; in differences of the fitting procedures, e.g., the way a quasi-continuum is adjusted; in differences of models, e.g., between LTE and non-LTE or between the individual versions of the line broadening theory; and in physical differences as the unrecognized presence of trace metals and their levitation (Barstow et al. 2003; Bohlin 2000; Chayer et al. 1995; Napiwotzki 1997; Schuh et al. 2002). Clearly, the extremely good counting statistics in the spectra of HZ43 A in all spectral ranges are not the only error source. The error ellipses in Fig. 3 denote our result for HZ43 A based on the interpretation of the Chandra soft X-ray spectrum. This result takes systematic errors partly into account and is described below.

\section{Results}

Model fits to the individual Chandra LETG+HRC spectra using the nominal (November 2004) first order effective areas $A^{0}$ 
and the nominal efficiencies of the higher orders relative to the first order yield marginally acceptable reduced $\chi_{v}^{2}=1.45$ and 2.04 for RX J1856 and Sirius B, respectively, but an enormous $\chi_{v}^{2}=32.40$ for HZ43 A. Following suggestions by others (e.g. Braje \& Romani 2002; Drake et al. 2002; Pease et al. 2000, 2003), we interprete these deficiencies as caused by the remaining errors in $A^{0}$ and search for a way to remove these defects. To this end, we include both hot white dwarfs in a simultaneous fit together with the neutron star RX J1856, which allows us to tie HZ43 A to the flux level of the latter and to the better defined effective areas at $\lambda<40 \AA$. In what follows, we discuss the normalizations of the model spectra and the treatment of the interstellar absorption which is of particular importance for RX J1856 and subsequently describe the combined fit.

\subsection{Visual and EUV fluxes}

We require that our incident spectra comply with the findings in other wavelength regions. For HZ43 A, we adopt the visual magnitude $V=12.909$ (Bohlin 2000), or equivalently adjust the model to the HST/STIS spectral flux at $5450 \AA$, $f_{5450}=2.558 \times 10^{-14} \mathrm{erg} \mathrm{cm}^{-2} \mathrm{~s}^{-1} \AA^{-1}$. In order to appropriately fix the interstellar absorbing hydrogen column density, we adopt a mean flux in the 475-495 $\AA$ region of $f_{485}=1.84 \times 10^{-12} \mathrm{erg} \mathrm{cm}^{-2} \mathrm{~s}^{-1} \AA^{-1}$, which is the spectral flux of the long-wavelength EUVE spectrum used by Wolff et al. (1999) and slightly exceeds that of Dupuis et al. (1995). For Sirius B, we adopt the $4600 \AA$ HST/STIS flux $f_{4600}=$ $2.860 \times 10^{-12} \mathrm{erg} \mathrm{cm}^{-2} \mathrm{~s}^{-1} \AA^{-1}$ taken from the carefully calibrated G430L HST/STIS spectrum of Barstow et al. (2005) which puts us on his optical flux scale. The mean 475-495 $E U V E$ flux of Sirius B, $\sim 6 \times 10^{-15} \mathrm{erg} \mathrm{cm}^{-2} \mathrm{~s}^{-1} \AA^{-1}$, has a huge error, but a flux of this order is consistent with the interstellar neutral hydrogen column density reported by Hébrard et al. $(1999), N_{\mathrm{HI}}=(6.5 \pm 2.0) \times 10^{17} \mathrm{~cm}^{-2}$. For RX J1856, we use the visual flux determined by van Kerkwijk \& Kulkarni (2001), $f_{5000}=2.96 \times 10^{-19} \mathrm{erg} \mathrm{cm}^{-2} \mathrm{~s}^{-1} \AA^{-1}$ at $5000 \AA$, and account for extinction using $A_{\mathrm{V}}=N_{\mathrm{HI}} /\left(1.87 \times 10^{21} x_{\mathrm{H}} \mathrm{cm}^{-2}\right)$ (Clayton et al. 2003), where $N_{\mathrm{HI}}$ is the fitted interstellar column density of atomic hydrogen in $\mathrm{cm}^{-2}$ and $x_{\mathrm{H}}$ is the ionization fraction of hydrogen.

\subsection{Interstellar photoelectric absorption}

The strength of the interstellar carbon K-shell absorption edge in the spectrum of RX J1856 is central to our discussion and requires that we discuss this aspect to some detail. We note that the application of the nominal LETG+HRC effective areas $A^{0}$ yields an unphysical carbon jump of the RX J1856 spectrum in emission, which corresponds to a negative carbon abundance $a(C)$. The appropriate treatment of carbon absorption allows us to fix the correction to the LETG+HRC effective area on the longwavelength side of the carbon jump. This is the first step in our approach to an internally consistent set of photon spectra for our three stars.

In treating interstellar photoelectric absorption, we largely follow Wilms et al. (2000). We use the cross-sections of hydrogenic ions as given by the analytic formula from Spitzer's textbook, the cross sections of Yan et al. (1998) for neutral helium, and the cross sections of Balucinska-Church \& McCammon (1992) and Verner \& Yakovlev (1995) as implemented in the most recent version of $\mathrm{XSPEC}^{4}$ for the heavier elements. For the short lines of sight to the objects considered here, we neglect molecules. We use the protosolar abundances of Lodders (2003) as representative of cosmic abundances, except for carbon which is discussed below, and consider the reduced effective cross-sections of atoms condensed in dust grains. The solar and cosmic abundance of carbon has long been considered to be around $400 \mathrm{ppm}$ (e.g. Holweger 2001) ${ }^{5}$, but Allende Prieto et al. (2002) re-determined the photospheric solar abundance to be $245 \mathrm{ppm}$, while Lodders (2003) suggested a protosolar abundance of $288 \pm 30 \mathrm{ppm}$ which is based on Allende Prieto's photospheric value and accounts for some elemental settling in the Sun. Measurements of the gaseous carbon abundance in the tenuous warm interstellar medium yield $\mathrm{C} / \mathrm{H} \simeq 140-161 \mathrm{ppm}$ (Cardelli et al. 1996; Sofia et al. 2004). There is a dispute about how much carbon is needed to explain the extinction properties of dust grains. Synthesis of the extinction curve for translucent lines of sight in terms of the properties of graphite, amorphous carbonaceous grains, and silicate grains suggests that a minimum of $180 \mathrm{ppm}$, and more typically $250 \mathrm{ppm}$, of carbon is needed to form grains with the observed properties (Clayton et al. 2003; Zubko et al. 2004), which suggests a carbon abundance in the general interstellar medium somewhat larger than protosolar. The earlier proposition of an interstellar carbon abundance lower than solar photospheric (e.g. Wilms et al. 2000) has become unlikely with the reduction of the solar value. We generously condense the available information into a range of the total interstellar carbon abundance of $320 \pm 80 \mathrm{ppm}$ of which $\sim 60 \%$ is in dust grains. The size distribution of the grains is a subject of dispute, too, since the ones deduced from the extinction curves in different directions (e.g. Clayton et al. 2003; Zubko et al. 2004) differ from that of grains of interstellar origin picked up in interplanetary space (Frisch et al. 1999). Larger grains produce less extinction and will be less efficient X-ray absorbers. We give preference to the size distribution derived from extinction curves and use a MRN distribution (Mathis et al. 1977; Clayton et al. 2003) with a cutoff at a maximum grain radius of $0.5 \mu \mathrm{m}$ as our best estimate to reproduce the effects of carbonaceous grains. The influence of this parameter on our results is moderate.

We fix the degrees of ionization of the interstellar hydrogen and helium along the line of sight to our three stars. For HZ43 A, we use $x_{\mathrm{H}}=0.10$ and $x_{\mathrm{He}}=0.40$ (Wolff et al. 1999). For the other two stars, we adopt $x_{\mathrm{H}}=0.25$ and $x_{\mathrm{He}}=0.40$, as determined from pickup ions of local interstellar origin and other studies (Slavin \& Frisch 2002; Gloeckler \& Geiss 2004). Doubly ionized helium is disregarded following Slavin \& Frisch (2002).

\subsection{The LETG+HRC spectrum of RXJ1856}

We followed Burwitz et al. (2003), Braje \& Romani (2002), and Pons et al. (2002) in fitting the observed spectrum of RX J1856 from the optical to the X-ray regime by the sum of two blackbodies with temperatures $k T_{\text {spot }}$ and $k T_{\text {star }}$, of which the hotter one $\left(k T_{\text {spot }}\right)$ describes the X-ray emitting spot. We provisionally interprete the cooler component $\left(k T_{\text {star }}\right)$ as the emission from the

\footnotetext{
4 http://heasarc.gsfc.nasa.gov/docs/xanadu/xspec

5 We quote abundances in parts per million (ppm) relative to hydrogen. $\mathrm{C} / \mathrm{H}=245$ (400) $\mathrm{ppm}$ corresponds to a logarithmic abundance of 8.39 (8.60).
} 


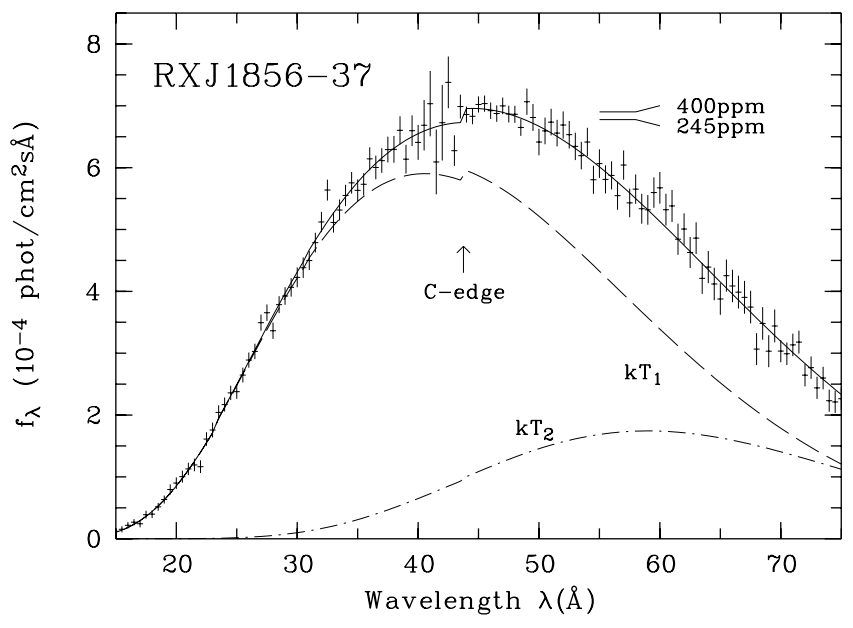

Fig. 4. Chandra LETG+HRC spectrum of RX J1856 binned to $0.5 \AA$, derived with the effective area at wavelengths longwards of the carbon edge corrected to fit an abundance of carbon in the interstellar medium of $a(C)=245-400 \mathrm{ppm}$ with $60 \%$ in dust grains. The two-temperature fit shown is for $320 \mathrm{ppm}$, with the upper level of the step indicated for 245 and $400 \mathrm{ppm}$, too. The fit has a $\chi^{2}=97.7$ for 115 d.o.f. (see Sect. 4.5). The model parameters are quoted in Table 2.

annulus representing the projected neutron star surface outside the $\operatorname{spot}^{6}$.

We fit the summed model spectrum for the first to the sixth diffraction orders to the Chandra LETG+HRC-S count rate spectra of RXJ1856 in the positive and negative dispersion directions. Figure 4 shows the corresponding summed photon spectrum, which allows to judge the strength of the interstellar carbon absorption edge more clearly than the count rate spectrum with its superposed effective area structure. The photon spectrum is created from the observed spectrum by subtracting the 2 nd to 6 th order model count rates from the data (in cts $\mathrm{s}^{-1} \AA^{-1}$ ) and dividing by the first-order effective area. The expected strength of the absorption edge is obtained if the effective areas shortwards of $43.7 \AA$ are accepted and longwards are moderately reduced. We perform fits to the wavelength region 15-39 $\AA$ and anchor the fit at long wavelengths where the higher orders dominate. This yields a data/model ratio different from unity in the 44.0-48.5 $\mathrm{A}$ range which we interprete as the mean factor $\alpha_{1}$ by which the effective area in this interval has to be adjusted in order to obtain a perfect fit with these wavelengths included. From best fits with $a(C)$ between 245 and $400 \mathrm{ppm}$ and $60 \%$ of the carbon in dust grains, we obtain $\alpha_{1} \equiv\langle A\rangle /\left\langle A^{0}\right\rangle=0.875 \pm 0.012$, where the angular brackets refer to averages over the $44-48.5 \AA$ interval. An additional and larger uncertainty arises from the errors in the fit parameters, notably $k T_{\text {star }}$, determined for an increase in $\chi^{2}$ by +1 over its minimum $\chi_{\min }^{2}=38.7$ for 44 d.o.f. in the $15-39 \AA$ range. Merging the two results yields $\alpha_{1}=0.871 \pm 0.038$. This factor applies within its error to both the negative and the positive dispersion directions. Figure 4 shows the derived summed

\footnotetext{
${ }^{6}$ Presently, the nature of the cool component is still disputed and so is the angular stellar radius derived from the blackbody assumption. A model in which an underlying blackbody flux is reprocessed in a thin surface layer of hydrogen can account for an enhancement of the optical flux relative to that at shorter wavelengths, but only by a factor of at most two (S. Dreizler, private communication) and, of course, it can not exceed the blackbody flux at its own temperature. A way to dissociate the optical component from the neutron star as a light source would be the presence of optical light emitting circumstellar matter.
}

spectrum of both sides using effective areas $A=\alpha(\lambda) A^{0}$ where the wavelength-dependent adjustment factor $\alpha$ is derived below and is forced to match $\alpha_{1}$ between 44 to $48.5 \AA$. Our photon spectrum is very similar to that presented by Drake et al. (2002, see their Fig. 1), who evidently used similarly adjusted effective areas. Our final fit with the adjusted areas extends over the spectral range $15-74 \AA$ and has $\chi^{2}=97.7$ for 115 d.o.f. This spectral range avoids the shortest wavelengths with low flux and the longest wavelengths where the higher orders become important and finally dominate.

\subsection{Simultaneous fit to the spectra of several stars}

\subsubsection{General approach}

The method for the determination of the area correction function $\alpha(\lambda)$ is based on the simultaneous fit to $n \geq 2$ stars with $N$ data points each, centered at wavelengths $\lambda_{1}$ to $\lambda_{N}$. Because of a lack of suitable calibrators, we apply the method for $n=2$ only. The standard $\chi^{2}$ which expresses the differences between the data $y_{i j}$ with statistical errors $\sigma_{i j}$ and the model contributions $m_{i j k}$ in orders $k=1$ to 6 (all in cts s${ }^{-1} \AA^{-1}$ ) is

$\chi^{2}=\sum_{j=1}^{n} \sum_{i=1}^{N} \frac{\left(y_{i j}-\sum_{k=1}^{6} m_{i j k}\right)^{2}}{\sigma_{i j}^{2}}$

with orders higher than $k=6$ entirely negligible for the objects considered. As usual, the model contributions are calculated as

$m_{i j k}=\frac{1}{k} f_{j}\left(\frac{\lambda_{i}}{k}\right) \alpha\left(\frac{\lambda_{i}}{k}\right) A^{0}\left(\frac{\lambda_{i}}{k}\right) \varepsilon_{k}\left(\frac{\lambda_{i}}{k}\right)=\alpha\left(\frac{\lambda_{i}}{k}\right) m_{i j k}^{0}$

where $f_{j}\left(\lambda_{i}\right)$ is the incident spectral flux of star $j$ in photons $\mathrm{cm}^{-2} \mathrm{~s}^{-1} \AA^{-1}, \varepsilon_{k}\left(\lambda_{i}\right)$ the efficiency of the $k$ th order relative to the first order, and $m_{i j k}^{0}$ are the model contributions calculated with the nominal effective areas. We correct the observed count rates for the respective model contributions in the second to sixth order to yield $y_{i j}^{\prime}=y_{i j}-\sum_{k=2}^{6} \alpha\left(\lambda_{i} / k\right) m_{i j k}^{0}$. Since $\alpha\left(\lambda_{i} / k\right)$ is involved, the transformation requires, in principle, an iterative approach. While this is easily implemented in the iterative procedure to minimize $\chi^{2}$, the influence of this procedure on the derivation of the $\alpha_{i}=\alpha\left(\lambda_{i}\right)$ is minute because (i) the adjusted areas affect the second and third orders only at $\lambda>88 \AA$ and $\lambda>132 \AA$, respectively, (ii) RX J1856 is not affected because we use its data only at $\lambda<74 \AA$ (see below), and (iii) the contributions of the higher orders to the spectra of HZ43 A and Sirius B are close to negligible. Hence the determination of the $\alpha_{i}$ is straightforward. We substitute the $y_{i j}$ in Eq. (1) by the $y_{i j}^{\prime}$, form the first-order count rate ratio of data vs. nominal model $r_{i j}=y_{i j}^{\prime} / m_{i j 1}^{0}$ with errors $\rho_{i j}=\sigma_{i j} / m_{i j 1}^{0}$, and find that the $\alpha_{i}$ are determined by minimizing the differences between the individual ratios $r_{i j}$,

$\chi^{2}=\sum_{j=1}^{n} \sum_{i=1}^{N} \frac{\left(y_{i j}^{\prime}-\alpha_{i} m_{i j 1}^{0}\right)^{2}}{\sigma_{i}^{2}}=\sum_{j=1}^{n} \sum_{i=1}^{N} \frac{\left(r_{i j}-\alpha_{i}\right)^{2}}{\rho_{i j}^{2}}$

For $n=2$ stars, we compute $\alpha_{i}$ as the weighted mean of $r_{i 1}$ and $r_{i 2}$,

$\alpha_{i}=\frac{\rho_{i 2}^{2} r_{i 1}+\rho_{i 1}^{2} r_{i 2}}{\rho_{i 1}^{2}+\rho_{i 2}^{2}}$, 
and obtain the final form of the $\chi^{2}$ to be minimized as

$\chi^{2}=\sum_{i=1}^{N} \frac{\left(r_{i 1}-r_{i 2}\right)^{2}}{\rho_{i 1}^{2}+\rho_{i 2}^{2}}$.

The expectation value of $\chi^{2}$ of Eq. (5) is $N-p$, since with $2 N$ data points we determine $p$ parameters in the models $m_{1}$ and $m_{2}$ and $N$ values of $\alpha_{i}$. The approach becomes insensitive to the $\alpha\left(\lambda_{i}\right)$ if the contributions of the higher orders at $\lambda_{i}$ become large, because the relative errors of the $y_{i j}^{\prime}$ increase correspondingly. Minimizing $\chi^{2}$ with the $\alpha_{i}$ included may only be marginally stable if the parameters allow a bulging deformation of both model spectra and a runaway of the $\alpha_{i}$. The white dwarf models are resistant against such deformation, however, and $\alpha\left(\lambda_{i}\right)$ is, furthermore, fixed to $\alpha_{1}=0.87$ in the interval $44-48.5 \AA$. In our application of the method, the fitted spectra of HZ43 A and RX J1856 are tied down in the optical and at $\sim 46 \AA$, while the spectrum of Sirius B is tied to HZ43 A.

We have opted to fit the sum of the model contributions for the positive and negative dispersion directions to the sum of the respective observed spectra. The so-defined model spectrum allows us to derive the combined correction function $\alpha$ from the combined observed spectrum as well as the individual $\alpha_{\text {neg }}$ and $\alpha_{\text {pos }}$ from the observed spectra for the negative and positive dispersion directions, respectively. The combined $\alpha$ is the areaweighted mean of $\alpha_{\text {neg }}$ and $\alpha_{\text {pos }}$,

$\alpha A^{0}=\alpha_{\mathrm{neg}} A_{\mathrm{neg}}^{0}+\alpha_{\mathrm{pos}} A_{\mathrm{pos}}^{0}$

with $A^{0}=A_{\text {neg }}^{0}+A_{\text {pos }}^{0}$. Multiplication of the nominal area with the respective $\alpha$ yields the adjusted areas $A_{\text {neg }}=\alpha_{\text {neg }} A_{\text {neg }}^{0}, A_{\text {pos }}=$ $\alpha_{\mathrm{pos}} A_{\mathrm{pos}}^{0}$, and $A=\alpha A^{0}$.

\subsubsection{Combined fit to RXJ1856, HZ43 A, and Sirius B}

The number of stars with sufficiently well-known incident spectra is extremely restricted. We fit, therefore, RX J1856 alone for $15 \leq \lambda<39 \AA$, RXJ1856 and HZ43 A simultaneously for $44 \leq \lambda<74 \AA$, and Sirius B and HZ43 A simultaneously for $74 \leq \lambda \leq 160 \AA$. We calculated a grand $\chi^{2}$ for the combined fit of the three models to the three observed spectra with 518 data points including three points in the visual and the two $485 \AA$ fluxes of HZ43 A and Sirius B (the latter chosen to reproduce the Hébrard et al. (1999) value of $N_{\mathrm{HI}}$ within its error). The number of free parameters includes the 12 listed in Table 2 plus the 232 values $\alpha_{i}$. Hence, there are 274 d.o.f. Figure 5 displays the results of the simultaneous fit to the three stars and Table 2 gives the best-fit parameters. The quoted $1-\sigma$ errors are derived from variation of the respective parameter with all other parameters kept free and refer to $\Delta \chi^{2}=+1$. The only parameter kept fixed in the fit was the gravity of Sirius B, $\log g=8.6$, which is reasonably well determined from the angular radius, the Hipparcos distance and the gravitational redshift (Barstow et al. 2005; Holberg et al. 1998). The grand $\chi^{2}$ for the simultaneous fit is 282.9 for 274 d.o.f. $\left(\chi_{v}^{2}=1.03\right)$ which is close to perfect.

The self-consistently determined multiplicative correction function $\alpha\left(\lambda_{i}\right)=A / A^{0}$ for the sum of negative and positive dispersion orders is shown at the top of Fig. 5. It converts the nominal LETG+HRC-S effective area $A^{0}$ of the November 2004 release to the area $A$ used in this paper and is valid for the best-fit $\log g=7.90$ of HZ43 A. It has been smoothed with a box car over three wavelength bins (i.e., a total width of $1.5 \AA$ ) in order
Table 2. Parameters of HZ43 A, Sirius B, and RX J1856 based on the simultaneous fit of our model spectra to the LETG+HRC count rate spectra in the wavelength intervals given. The quoted $1-\sigma\left(\Delta \chi^{2}=+1\right)$ errors are correlated and derived from fits with the other parameters for each object kept free. The letter $f$ indicates: fixed.

\begin{tabular}{lc}
\hline \hline Parameter & Value \pm Error \\
\hline (a) $H Z 43 A(\lambda=45-160 \AA)$ & \\
$T_{\text {eff }}(\mathrm{K})$ & $51126 \pm 660$ \\
$\log g$ & $7.90 \pm 0.08$ \\
$R^{2} / d^{2}\left(10^{-23}\right)$ & $8.011 \pm 0.038$ \\
$N_{\mathrm{HI}}\left(10^{17} \mathrm{~cm}^{-2}\right)$ & \\
(b) $\operatorname{Sirius} B(\lambda=74-160 \AA)$ & \\
$T_{\text {eff }}(\mathrm{K})$ & $24923 \pm 115$ \\
$\log g$ & $8.6 f^{1}$ \\
$R^{2} / d^{2}\left(10^{-21}\right)$ & $4.877 \pm 0.036$ \\
$N_{\mathrm{HI}}\left(10^{17} \mathrm{~cm}^{-2}\right)$ & $6.5 \pm 2.02$ \\
(c) $R X J 1856(\lambda=15-74 \AA)$ & \\
$k T_{\text {spot }}(\mathrm{eV})$ & $62.83 \pm 0.41$ \\
$k T_{\text {star }}(\mathrm{eV})$ & $32.26 \pm 0.72$ \\
$R_{1} / d(\mathrm{~km} / \mathrm{pc})$ & $0.0378 \pm 0.0003$ \\
$R_{2} / d(\mathrm{~km} / \mathrm{pc})$ & $0.1371 \pm 0.0010$ \\
$N_{\mathrm{HI}}\left(10^{20} \mathrm{~cm}{ }^{-2}\right)$ & $1.10 \pm 0.03$ \\
\hline
\end{tabular}

${ }^{1}$ Based on Barstow et al. (2005); Holberg et al. (1998).

${ }^{2}$ Hébrard et al. (1999). Our fit is required to stay within the $1-\sigma$ error.

to reduce the influence of statistical fluctuations in the individual spectra. The correction is consistent with unity at $\lambda<44 \AA$, but reaches a low of about 0.77 at $65 \AA$, consistent with Pease's et al. (2003) expectation that the error in the 60-80 $\AA$ range might reach $25 \%$. At longer wavelengths, $\alpha\left(\lambda_{i}\right)$ moves closer to unity again. For $\log g$ values of HZ43 A other than 7.90, the whole curve moves up and down a bit with a pivot point at $\alpha(46 \AA)=0.87 \pm 0.04$. In order to mark the extent of this variation, we have included the systematic errors at 46, 70, 90, and $125 \AA$ with an upper (lower) cross bar signifying the location of $\alpha\left(\lambda_{i}\right)$ for $\alpha_{1}=0.91(0.83)$ and $\log g=8.00$ (7.80) for HZ43 A, respectively. None of the other parameters has a similarly large influence. In summary, we conclude that the necessary adjustment to the LETG+HRC-S effective area relative to that at $\lambda \lesssim 40 \AA$ is pronounced in the $50-80 \AA$ region, but small longwards of $85 \AA$. Its systematic uncertainty is $\pm 5 \%$ at $\lambda \gtrsim 80 \AA$ from the uncertainty in $\log g$ of HZ43 A and another $5 \%$ at all wavelengths $\lambda \gtrsim 44 \AA$ from the error in $\alpha_{1}$. The fact that $\log g$ of Sirius B is better defined than that of HZ43 A does not help because the spectrum of Sirius B does not extend to $44 \AA$ where it could be tied down.

Our derived areas for the negative and positive dispersion directions, $A_{\text {neg }}=\alpha_{\text {neg }} A_{\text {neg }}^{0}$ and $A_{\text {pos }}=\alpha_{\text {pos }} A_{\text {pos }}^{0}$, are close to the SRON areas suggested previously by J. Kaastra (Pease et al. 2000; and J. Kaastra, private communication) between 44 and $80 \AA$ and close to the nominal CXC areas at longer wavelengths. Our results are provided in Tables A.1 to A. $3^{7}$ for the preferred $\log g$ of HZ43 A of 7.90 as well as for 7.80 and 8.00, indicating the systematic errors of our fit. The following systematic uncertainties are not included in these tables: (i) the normalization uncertainty at $\sim 46 \AA$ as expressed by $\alpha_{1}=0.87 \pm 0.04$; (ii) the possible degradation in the HRC response with time; and (iii) the absolute error in the nominal areas $A^{0}$ at short wavelengths.

7 Tables A.1 to A.3 are only available electronically at CDS. 


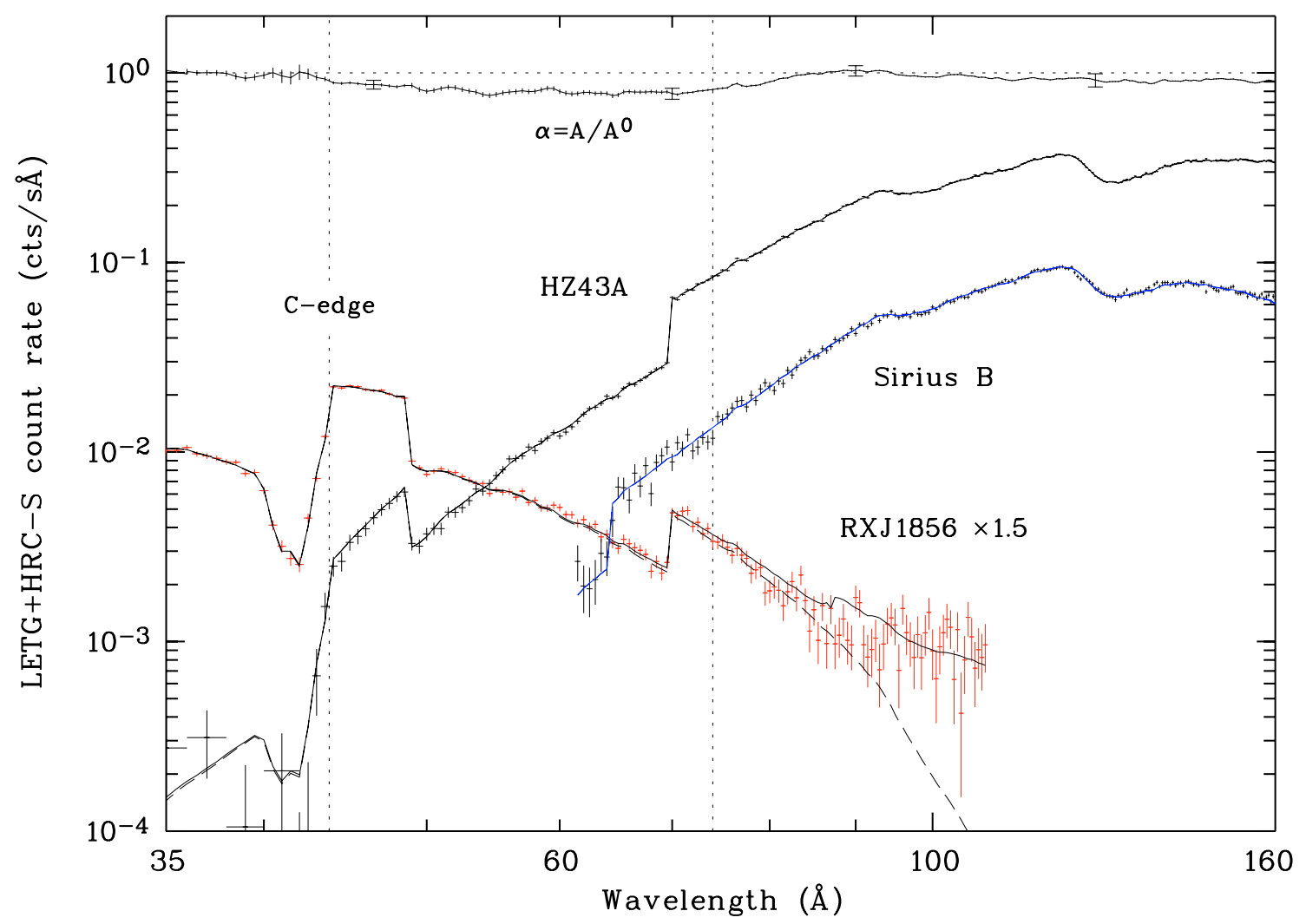

Fig. 5. Simultaneous fit of RX J1856, HZ43 A, and Sirius B in the wavelength ranges marked by vertical dotted lines (see Sect. 4.4.2). The LETG spectra binned to $0.5 \AA$ are shown as data points, the corresponding best-fit models as solid curves, and the first-order contributions as dashed curves. The area correction function $\alpha$ is shown at the top. It converts the nominal LETG+HRC-S first-order effective area $A^{0}$ of the November 2004 release into the adjusted area $A$ used in this paper. Systematic uncertainties in $\alpha$ are indicated by error bars at $46,70,90$, and $125 \AA$. The steps in the count rate spectra of HZ43 A and RXJ1856 at 49 and $69 \AA$ A result from the detector gaps. Sirius B was observed off axis and its gaps are located differently (see text).

As noted above, our empirically adjusted effective areas are strictly valid only for the epoch JD 2452130 . The possible variation of the HRC sensitivity since the begin of the mission is small, however, 0.015 in $\alpha\left(\lambda_{i}\right)$ at long wavelengths and possibly as much as 0.050 shortwards of about $50 \AA$ (Fig. 1). Nevertheless, the possibility of a slow degradation in $A_{\text {neg }}$ and $A_{\text {pos }}$ should be kept in mind: it ultimately limits the accuracy of parameters determined from fits to the long wavelength sections of LETG+HRC spectra.

\subsubsection{The photon spectra of $H Z 43 A$, Sirius B, and RXJ1856}

The incident photon spectra of the three stars fitted simultaneously are obtained by dividing the count rate spectra of Fig. 5 by our effective areas. Their errors are dominated by the systematic errors as discussed in the last section. Figure 6 shows the resulting photon spectra with the systematic uncertainties indicated again by additional error bars at 60 and $125 \AA$. The spectral fluxes at selected wavelengths are listed in Table 3 together with their systematic errors arising from $\alpha$ in percent given in brackets. The complete spectra are provided in Tables A.4 to A.6. The systematic errors listed near the end of the last Section under (ii) and (iii) are not included. The bottom three lines of Table 3 contains the optical fluxes to which the spectra were normalized. The fourth line from the bottom gives the predicted fluxes at $1300 \AA$. In the case of HZ43 A, the quoted flux agrees to better than $0.3 \%$ with that of the HST/STIS spectrum. For Sirius B,

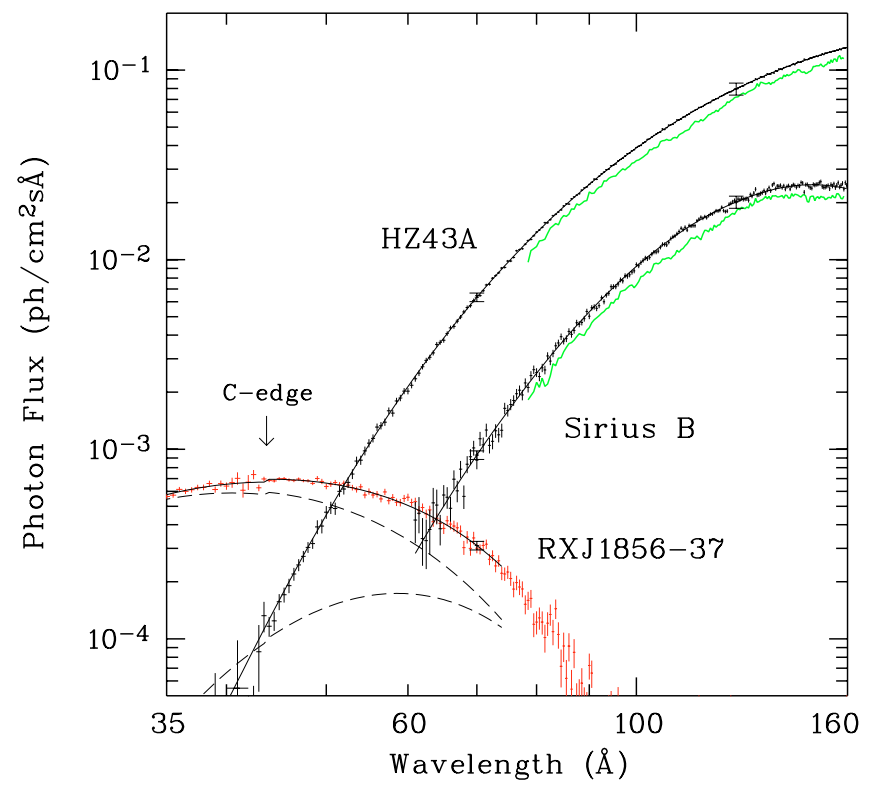

Fig. 6. Photon spectra of RX J1856, HZ43 A, and Sirius B based on our analysis of the LETG+HRC-S observations (data points) and best fit model spectra with parameters as given in Table 2 (solid curves). The spectrum of RXJ1856 is the same as in Fig. 4 with the two blackbody components shown separately as dashed curves. Longwards of $78 \AA$, we have included the EUVE spectra of the two white dwarfs (green/gray curves). 
Table 3. Soft X-ray fluxes as defined by the best-fit absorbed photon spectra in Fig. 6. The numbers in brackets are the systematic errors of the LETG+HRC-S area correction in percent (see text).

\begin{tabular}{|c|c|c|c|}
\hline $\begin{array}{c}\lambda \\
(\AA) \\
(\AA)\end{array}$ & HZ43 A & \multicolumn{2}{|c|}{ photons $\mathrm{cm}^{-2} \mathrm{~s}^{-1} \AA^{-1}$} \\
\hline $\begin{array}{r}20 \\
30 \\
40 \\
43 \\
44 \\
48 \\
60 \\
70 \\
80 \\
90 \\
100 \\
125 \\
160\end{array}$ & $\begin{array}{l}3.01 \times 10^{-4} \quad(5) \\
2.14 \times 10^{-3}(5) \\
6.33 \times 10^{-3}(5) \\
1.39 \times 10^{-2}(6) \\
2.49 \times 10^{-2}(6) \\
3.89 \times 10^{-2}(6) \\
7.96 \times 10^{-2}(7) \\
1.31 \times 10^{-1}(7)\end{array}$ & $\begin{array}{ll}2.49 \times 10^{-4} & (6) \\
9.57 \times 10^{-4} & (6) \\
2.58 \times 10^{-3} & (6) \\
5.43 \times 10^{-3} & (6) \\
9.47 \times 10^{-3} & (6) \\
2.10 \times 10^{-2} & (7) \\
2.55 \times 10^{-2} & (7)\end{array}$ & $\begin{array}{ll}8.54 \times 10^{-5} & (5) \\
4.32 \times 10^{-4} & (5) \\
6.54 \times 10^{-4} & (5) \\
6.70 \times 10^{-4} & (5) \\
6.94 \times 10^{-4} & (5) \\
6.81 \times 10^{-4} & (5) \\
5.06 \times 10^{-4} & (7) \\
3.11 \times 10^{-4} & (7)\end{array}$ \\
\hline 160 & \multicolumn{3}{|c|}{$\operatorname{erg~cm}{ }^{-2} \mathrm{~s}^{-1} \AA^{-1}$} \\
\hline $\begin{array}{l}1300 \\
4600 \\
5000 \\
5450\end{array}$ & $4.052 \times 10^{-12}$ & $\begin{array}{l}1.258 \times 10^{-10} \\
2.860 \times 10^{-12}\end{array}$ & $\begin{array}{l}5.07 \times 10^{-172} \\
2.96 \times 10^{-193}\end{array}$ \\
\hline
\end{tabular}

${ }^{1}$ The Compton effect may depress this flux by $\sim 1 \%$.

${ }^{2}$ The unabsorbed flux is $6.27 \times 10^{-17} \mathrm{erg} \mathrm{cm}^{-2} \mathrm{~s}^{-1} \AA^{-1}$.

${ }^{3}$ The unabsorbed flux is $3.18 \times 10^{-19} \mathrm{erg} \mathrm{cm}^{-2} \mathrm{~s}^{-1} \AA^{-1}$.

the $1300 \AA$ model flux agrees with that predicted by Vennes \& Dupuis (2002) and both exceed the IUE flux which is known to be too low (Massa \& Fitzpatrick 2000).

\subsection{Results for individual stars}

We now discuss the results for the individual stars taking the systematic errors as far as possible into account. These fits are characterized by $\chi^{2}=97.7$ for RX J1856 in the interval $15-74 \AA$ with 115 d.o.f., $\chi^{2}=183.9$ for HZ43 A in $45.5-160 \AA$ with 229 d.o.f., and $\chi^{2}=209.4$ for Sirius B in $61.5-160 \AA$ with 197 d.o.f. All fits are substantially improved over those without the area correction and can all be considered "good" in a statistical sense. This result is not subject to the binning chosen as in previous fits to the LETG+HRC spectrum of RX J1856 (e.g. Braje \& Romani 2002). The combined $\chi^{2}$ for HZ43 A and Sirius B is 393.3 for 426 d.o.f. and the slight disparity between the two stars results form the better statistics of HZ43 A which drives the fit.

\subsubsection{HZ43 A}

The errors of the individual parameters listed in Table 2 are, in part, highly correlated. This is particularly true of $T_{\text {eff }}$ and $\log g$ for HZ43 A. The corresponding error ellipses for the $68 \%$, $90 \%$ and $95 \%$ confidence limits are overplotted in Fig. 3. Gratifyingly, the best-fit values are in good agreement with results obtained from the optical and ultraviolet regimes. The large extent along the major axis of the ellipses reflects the correlation between the results for $T_{\text {eff }}$ and $\log g$. The soft X-ray flux stays essentially constant along the major axis and the narrow width in the perpendicular direction signifies the rapid deterioration of the fit if the model does not meet the observed flux. Hence, the soft X-ray flux in Table 3 is better defined by the fit than either $T_{\text {eff }}$

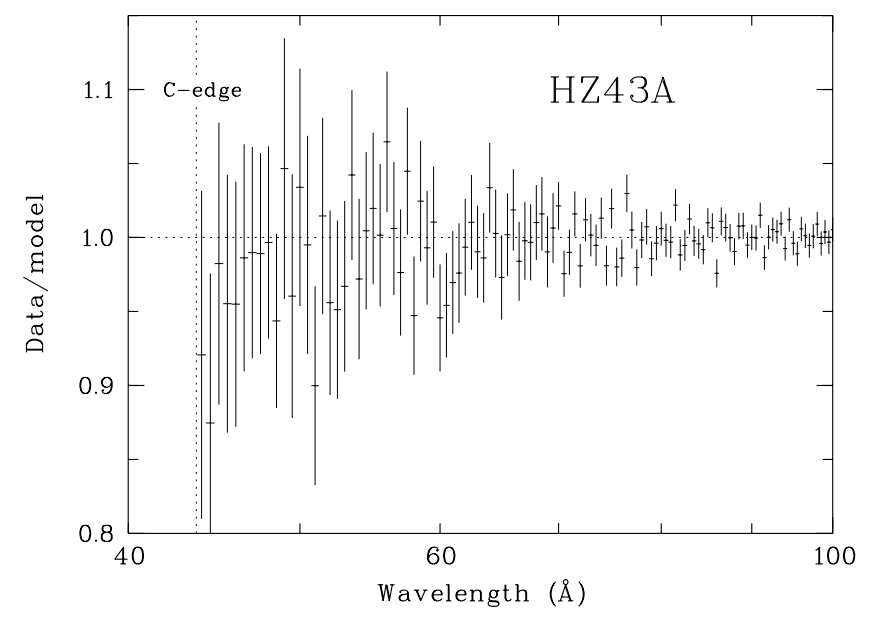

Fig. 7. Data vs. model flux ratio of the spectrum of HZ43 A for $\lambda>$ $43.5 \AA$. The maximum depression of the spectrum by the Compton effect near the carbon K-edge at $43.7 \AA$ is at the percent level.

or $\log g$. Parameter combinations like those in the lower left of Fig. 3 can safely be excluded since they yield much too low a flux, parameter combinations in the upper right would yield too large a flux. This is an important result for the future calibration of instruments sensitive in this spectral regime: it is more appropriate to quote soft X-ray fluxes than an effective temperature.

The angular radius $R / d$ of HZ43 A fixes the distance $d$ for a given radius $R$. Adopting Wood (1995) stellar models with a thick hydrogen envelope provides an independent relation and yields $M=0.64 \pm 0.05 M_{\odot}, R=(1.04 \pm 0.08) \times 10^{9} \mathrm{~cm}$, and $d=61.3 \pm 5.5 \mathrm{pc}$ with errors which account for the systematic uncertainty due to the full gravity range $\log g=7.90 \pm 0.10$. The gravitational redshift predicted for these parameters is $v_{\mathrm{g}}=$ $28 \pm 4 \mathrm{~km} \mathrm{~s}^{-1}$. The observed $v_{\mathrm{g}}=30.1 \pm 1.5 \mathrm{~km} \mathrm{~s}^{-1}$ (Reid 1996) suggests the slightly more restricted range $M=0.67 \pm$ $0.02 M_{\odot}$ (Dupuis et al. 1998), with $R=(9.92 \pm 0.34) \times 10^{8} \mathrm{~cm}$, $\log g=7.95 \pm 0.04$, and $d=59 \pm 2$ pc ( $1-\sigma$ errors $)$. Both distance values are consistent with the trigonometric parallax $\pi_{\mathrm{abs}}=15.3 \pm 2.9$ mas $\left(d=65 \pm_{10}^{15} \mathrm{pc}\right)$ from the Yale Catalog of Trigonometric Parallaxes (van Altena et al. 2001).

In principle, the LETG+HRC data of HZ43 A can help to decide whether the short-wavelength spectra of hot white dwarfs are steepened by the fact that electron scattering is no longer in the Thomson limit and must be correctly described by the Compton effect which degrades the energies of the scattered photons. In Fig. 7, we show the data/model ratio of the photon spectrum of HZ43 A from Fig. 6 for $\lambda \geq 44 \AA$. The data points are all more or less consistent with a ratio equal to unity: $1.0005 \pm 0.0014$ for $\lambda=65-100 \AA$; $0.995 \pm 0.011$ for $\lambda=49-58 \AA$; and $0.959 \pm 0.027$ for $\lambda=44-49 \AA$. The glitch in the ratio near $60 \AA$ may be a relic of differences between the positive and negative dispersion directions. We can not exclude an effect at the percent level given the remaining systematic errors, but the effect may as well be still smaller as suggested by Suleimanov et al. (2006).

\subsubsection{Sirius $B$}

The angular radius for Sirius B in Table 2 falls between the values quoted by Barstow et al. (2005) for the HST/STIS G430L 
and G750L spectra. At the Hipparcos distance of $2.637 \pm$ $0.011 \mathrm{pc}$, the radius of the white dwarf is $R=(5.683 \pm 0.019) \times$ $10^{8} \mathrm{~cm}=(8.165 \pm 0.028) \times 10^{-3} R_{\odot}$, where the error includes the systematic error in the LETG+HRC-S area correction and is insensitive to $\log g$ of Sirius B itself. Combined with the gravitational redshift of Barstow et al. (2005), $v_{\mathrm{gr}}=80.42 \pm 4.83 \mathrm{~km} \mathrm{~s}^{-1}$, we obtain a mass of $M=1.032 \pm 0.062 M_{\odot}$, consistent with the astrometric solution of Gatewood \& Gatewood (1978). The implied gravity $\log g=8.627 \pm 0.026$ slightly exceeds the spectroscopic value of Barstow et al. (2005), but stays within Holberg's et al. (1998) 1- $\sigma$ error. Conversely, the effective temperature of $24923 \pm 115 \mathrm{~K}$ (systematic error) falls below Barstow's spectroscopic value of $25193 \pm 37 \mathrm{~K}$ (statistical error) derived from a Balmer line fit to the HST/STIS spectrum. The possible systematic error quoted by Barstow et al. (2005) is $350 \mathrm{~K}$ and encompasses our result ${ }^{8}$. A spectrum calculated with our code for Barstow's $T_{\text {eff }}$ and $\log g$ adjusted to the same visual HST/STIS flux as above yields a predicted LETG+HRC spectral flux 14\% higher than our spectrum in Fig. 6, about twice its $\log g$-derived systematic error of $7 \%$.

\subsection{3. $\mathrm{RXJ1856}$}

The high quality $\left(\chi_{v}^{2}=0.87\right)$ of the two-blackbody fit to the LETG+HRC spectrum of RXJ1856 in Figs. 4 and 6 demonstrates that the deviation from this model is minute. The model is normalized to the extinction-corrected optical flux of van Kerkwijk \& Kulkarni (2001) and provides an almost perfect fit to all available data. Blackbody temperature and angular radius of the hotter component, $k T_{\text {spot }}=62.8 \pm 0.4 \mathrm{eV}$ and $0.0378 \mathrm{~km} \mathrm{pc}^{-1}$ (Table 2), agree well with previous determinations (Burwitz et al. 2001, 2003; Braje \& Romani 2002; Drake et al. 2002; Pons et al. 2002). The temperature $k T_{\text {star }}=32.3 \pm 0.7 \mathrm{eV}$ of the cooler component is determined here more accurately than before as a result of our elimination of some of the deficiencies in the LETGS effective area calibration at long wavelengths. The angular radius of this component (Table 2) refers to the outer radius of a blackbody-emitting annulus surrounding the hot spot. The quoted errors account for the systematic uncertainty in the area correction and indicate that the main error source for the radii still lies in the distance uncertainty. The excellence of the two-blackbody fit does not imply that RX J1856 actually radiates as a blackbody (see, e.g., the discussion in Kargaltsev et al. 2005). E.g., a graybody with its different limb darkening law would yield a different radius than the disk of uniform brightness. However, whatever the emission properties, the spectral flux integrated over the surface must nevertheless agree with that of the blackbody model within the statistical uncertainties of the $\mathrm{X}$-ray and optical/ultraviolet data. Hence, the blackbody fit is, in fact, quite restrictive. For the purpose of using RX J1856 as a soft X-ray calibrator the blackbody assumption is entirely satisfactory and need be replaced only if future observations reveal fine structure in the spectra not accounted for by this approximation. At present, we agree with (Braje \& Romani 2002) that there is no evidence for a more complex temperature distribution beyond the dichotomy of base and spot temperature.

\footnotetext{
${ }^{8}$ Note that our systematic error of $115 \mathrm{~K}$ does not include the uncertainty in the visual flux of Sirius B: a +0.01 mag change (1\% decrease in the adopted $4600 \AA$ flux) raises our value of the effective temperature of Sirius B by $14 \mathrm{~K}$ or $0.057 \%$. It is in the nature of our simultaneous fit that at the same time the effective temperature of HZ43 A is slightly reduced (by $0.050 \%$ ). The soft X-ray fluxes stay practically unchanged.
}

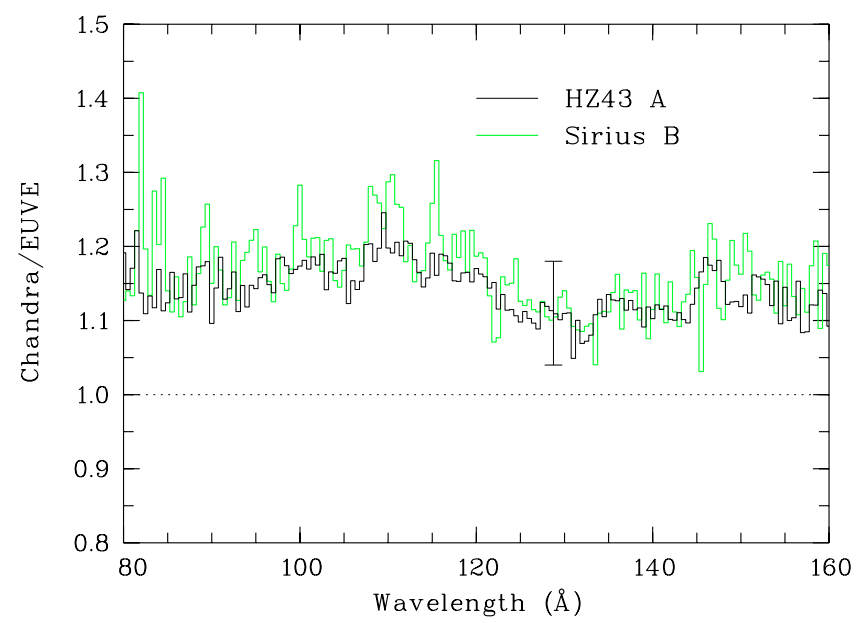

Fig. 8. Ratio of the soft X-ray fluxes in HZ43 A and Sirius B based on the Chandra LETG+HRC spectra analyzed here and the EUVE archive spectra listed in Table 1 . The results are valid for the calibration using $\log g(\mathrm{HZ} 43 \mathrm{~A})=7.90$ and would shift up or down by the systematic error shown at $128 \AA$ for $\log g$ lower or higher by 0.1 , respectively.

\subsection{Calibration of the EUVE SW spectrometer}

It is instructive to compare our photon spectra of HZ43 A and Sirius B with the short-wavelength spectra of these stars in the EUVE archive (Table 1). The mean EUVE spectra are included in Fig. 6 spectra and are seen to fall below the Chandra LETG+HRC spectra in our calibration. Figure 8 shows the ratios of Chandra vs. EUVE fluxes. They average 1.14 and 1.16 for HZ43 A and Sirius B, respectively. The systematic error of $7 \%$ from the uncertainty in $\log g$ of HZ43 A would shift both numbers up or down. This comparison suggests that the effective area used for the short-wavelength section of the EUVE archive spectra is too high by a factor of $1.15 \pm 0.07$ (systematic error). A similar discrepancy between their best-fit models and the EUVE short-wavelength fluxes was noted previously by Sing et al. (2002). Interestingly, they found agreement of the models with the EUVE fluxes at longer wavelengths, a result which has implicitely been adopted in our approach of determining the hydrogen column density by fitting the model spectrum to the EUVE long-wavelength flux at $485 \AA$. Our factor of 1.15 at the short wavelengths happens to be close to that by which the EUVE short-wavelength effective area determined on ground was corrected upward after an in-flight calibration using theoretical spectra of the white dwarfs HZ43 A, GD71, and GD153 (Boyd et al. 1994). In retrospect, that correction seems to have been unnecessary.

\subsection{ROSAT PSPC calibration}

We test the ROSAT PSPC calibration by folding the photon spectra of Fig. 6 through the PSPC detector response and compare them with the observed low-resolution PSPC spectra. We consider here the spectra of HZ43 A and Sirius B which were observed early in the mission ( $\mathrm{PV}$ and $\mathrm{AO}-1$ phases) and about two years into the mission (AO-2), before and after the change in high voltage which affected the detector response (see Sect. 2.3 and Table 1). We have selected two long observations of $\mathrm{HZ} 43 \mathrm{~A}$, one in the open configuration during the PV phase with PSPC-C and one with the boron filter in AO1 with PSPC-B. Sirius B was observed only once with the boron filter in $\mathrm{AO}-1$. We used the appropriate standard detector matrices 


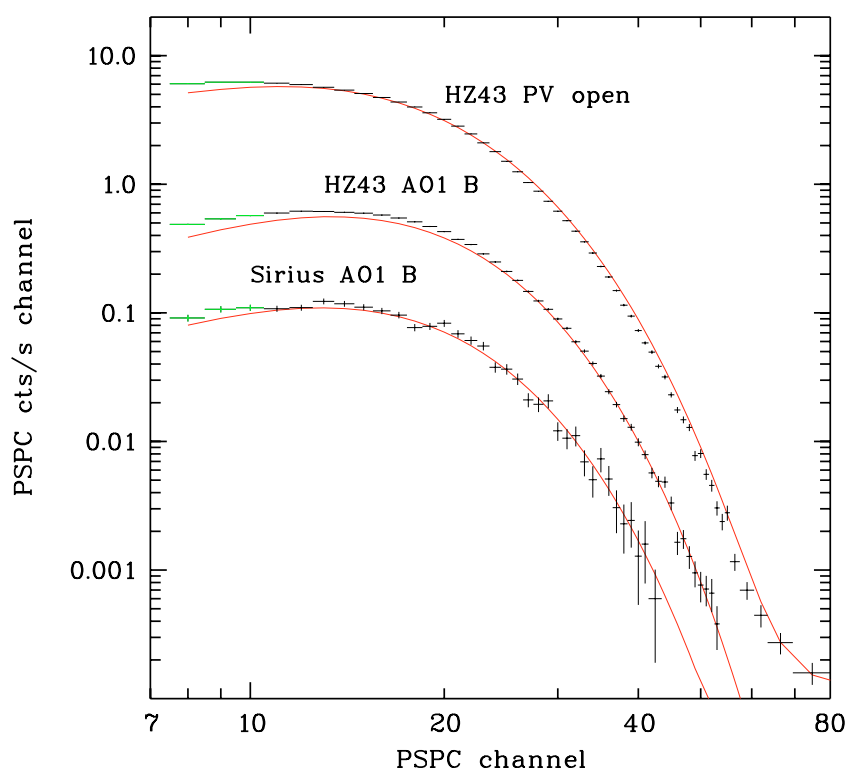

Fig. 9. ROSAT PSPC spectra of HZ43 A and Sirius B in either the open configuration or with boron filter (B). The abscissa is in channels with one channel corresponding to an apparent photon energy of $10 \mathrm{eV}$. The model spectra based on the fits to the LETGS observations are shown as solid curves, the PSPC observations as data points with statistical errors. HZ43 A possesses a bremsstrahlung component which commences at channel 65 and is included in the fit.

DRMPSPC-AO1 and DRMPSPC and applied no corrections for gain shifts (see Sect. 2.3). The resulting PSPC spectra are shown in Fig. 9 and reproduce the observations exceedingly well. The relevant integrated count rates for energy channels 11-60 are listed in Table 4. The errors given for the predicted count rates refer to the systematic uncertainty in our photon spectra and LETG+HRC-S area correction discussed above. The observed count rate of HZ43 A in the open configuration agrees perfectly with the prediction. Hence, we can safely conclude that the calibration of the PSPC is correct and the suggestions of a miscalibration by Napiwotzki et al. (1993), Jordan et al. (1994), and Wolff et al. $(1995,1996)$ are unfounded. Close inspection reveals some small discrepancies between prediction and observation which find ready explanations in a variety of small necessary corrections. In the uppermost spectrum in Fig. 9, the HZ43 observation in the open mode, the observed spectrum is slightly shifted to lower energy channels. This effect is caused by the gain depression by some $4 \%$ in the small central focal spot (Snowden et al. 2001). The observational "wobble" mode moves the source forth and back across this central depression and causes a mean gain reduction of about $1.5 \%$ which accounts for the observed shift. Of a different origin is the slight excess in count rate for the observations with boron filter (lower two spectra in Fig. 9). A probable explanation is provided by an actual thickness of the boron window about $4 \%$ lower than nominal.

The remaining small calibration uncertainties of the PSPC and the calibration of the high resolution imager (HRI) will be addressed elsewhere. All these effects are at the few percent level and provide no basis for the allegation of a miscalibration. In summary, the internal consistency of our results suggests that the PSPC yields a correct measurement of the soft X-ray flux and the ground calibration of the detector at soft X-ray energies is reliable to better than $10 \%$, as concluded also by Snowden et al. (1995). Given the large number of observations of all types
Table 4. Observed PSPC count rates in channels 11-60 and LETGSpredicted count rates based on the photon spectra in Fig. 6. "O" and "B" in Col. 3 refer to observations in the open configuration and with the boron filter, respectively.

\begin{tabular}{lccrrr}
\hline \hline \multirow{2}{*}{ Object } & Date & Filter & $\begin{array}{c}\text { Exp. } \\
\text { start }\end{array}$ & & \multicolumn{2}{c}{ Observed } & \multicolumn{1}{c}{ Predicted } \\
& (cts/s) & \multicolumn{1}{c}{ (cts/s) } \\
\hline HZ43 A & 900621 & O & 21.5 & $66.08 \pm 0.30$ & $65.38 \pm 2.70$ \\
& 910619 & B & 21.6 & $8.04 \pm 0.03$ & $7.35 \pm 0.28$ \\
Sirius B & 910315 & B & 3.3 & $1.43 \pm 0.03$ & $1.38 \pm 0.05$ \\
RX J1856 & 921111 & O & 6.3 & $3.51 \pm 0.03$ & $3.33 \pm 0.17$ \\
\hline
\end{tabular}

of celestial sources, both in the All-Sky Survey and in pointed observations, we judge this a reassuring statement of general interest. One can only speculate on the basis of the reports about a major miscalibration of the PSPC. The use of model spectra which provided perfect fits to observations in the optical and ultraviolet but lacked flux in the soft X-ray regime is a possibility.

In passing, we confirm that HZ43 A possesses a bremsstrahlung component with a temperature of $0.6 \mathrm{keV}$ and an emission measure of $3 \times 10^{52} \mathrm{~cm}^{-3}$, which is seen to commence near channel 65 in Fig. 9. It was previously reported by O'Dwyer et al. (2003) and probably originates from the secondary star. We have checked the zeroth order images of all individual LETGS observations for a signal at the position of the secondary (separation 3 arcsec, position angle $280^{\circ}$ ), but the point spread function of the white dwarf image still contributes a flux about an order of magnitude larger than expected for the secondary even if the position of the secondary is favorably located between the diffraction spikes. Hence, an origin of the hard X-rays from the secondary is plausible but remains unproven.

\section{Conclusions}

We have established the neutron star RX J1856 and the hot white dwarfs HZ43 A and Sirius B as standard stars in the soft X-ray regime by fitting the LETG+HRC spectra of all three stars simultaneously with the best available model spectra for the white dwarfs and a two-blackbody model for the neutron star. Our method ties the soft X-ray spectra of the two white dwarfs to that of the neutron star RX J1856 and thereby to be better calibrated effective areas of the Chandra LETG+HRC spectrometer shortwards of $40 \AA$. The spectrum of HZ43 A (and indirectly that of Sirius B) is thus fixed at soft X-ray and at optical wavelengths, a procedure which avoids the wagging-tail problem of previous calibration attempts using white dwarfs. Our second major result is the self-consistently determined correction to the LETG+HRC-S effective areas longwards of $44 \AA$. We find that the areas for both dispersion directions need a maximum correction of about $-23 \%$ at $65 \AA$ and are correct to within $\sim 10 \%$ at wavelengths exceeding $80 \AA$. These corrections are valid relative to the short-wavelength effective areas. The largest remaining uncertainty, which is not addressed here, lies in the absolute calibration of the LETG+HRC-S at short wavelengths. Our photospheric parameters of the three stars are in good agreement with those derived from independent optical/ultraviolet and soft $\mathrm{X}$-ray studies and, in part, improve on them. The soft X-ray flux of HZ43 A can be produced by a range of $T_{\text {eff }}-\log g$ combinations and is better defined than either of these two parameters.

We find that the calibration of the EUVE short-wavelength spectrometer differs from that of the Chandra LETG+HRC-S 
by about $15 \pm 7 \%$ in the sense that the original ground calibration seems to have been more adequate than the adopted in-flight calibration.

We demonstrate that the ground calibration of the ROSAT PSPC is correct to within a few percent and that previous reports of a major miscalibration are unfounded. The observed PSPC spectra of our three stars agree within a few percent with the LETGS-based soft X-ray spectra folded through the PSPC response. This internal consistency, in turn, supports our adjustment of the LETG+HRC-S calibration at long wavelengths and the basic correctness of the nominal calibration at short wavelengths.

Acknowledgements. This research was supported by the DLR under grant 50 OR 0201 (T.R.). We thank the referee Jay Holberg for helpful questions and comments, our colleagues from the ROSAT team and numerous colleagues from the general community for discussions, advice, and support, Frank Haberl for contributions in the early phase of this project, and Michael Freyberg for information on the calibration of the ROSAT PSPC. KB is indebted to Stefan Dreizler, Boris Gänsicke, Frederik Hessman, Klaus Reinsch, and Sonja Schuh for many discussions, and to Joachim Trümper for helpful comments and criticism.

\section{References}

Allende Prieto, C., Lambert, D. L., \& Asplund, M. 2002, ApJ, 573, L137 Balucinska-Church, M., \& McCammon, D. 1992, ApJ, 400, 699

Barstow, M. A., Holberg, J. B., \& Koester, D. 1995, MNRAS, 274, L31

Barstow, M. A., Holberg, J. B., Hubeny, I., et al. 2001, MNRAS, 328, 211

Barstow, M. A., Good, S. A., Burleigh, M. R., et al. 2003, MNRAS, 344, 562

Barstow, M. A., Bond, H. E., Holberg, J. B., et al. 2005, MNRAS, 362, 1134 Bohlin, R. C. 2000, AJ, 120, 437

Bohlin, R. C., Dickinson, M. E., \& Calzetti, D. 2001, AJ, 122, 2118

Boyd, W. T., Jelinsky, P. N., Finley, D. S., et al. 1994, Proc. SPIE, 2280, 280

Braje, T. M., \& Romani, R. W. 2002, ApJ, 580, 1043

Briel, U., et al. 1995, ROSAT User's Handbook, MPE

Brinkman, B. C., Gunsing, T., Kaastra, J. S., et al. 2000, Proc. SPIE, 4012, 81

Burwitz, V., Zavlin, V. E., Neuhäuser, R., et al. 2001, A\&A, 379, L35

Burwitz, V., Haberl, F., Neuhäuser, R., et al. 2003, A\&A, 399, 1109

Cardelli, J. A., Meyer, D. M., Jura, M., \& Savage, B. D. 1996, ApJ, 467, 334

Chayer, P., Vennes, S., Pradhan, A. K., et al. 1995, ApJ, 454, 429

Clayton, G. C., Wolff, M. J., Sofia, U. J., Gordon, K. D., \& Misselt, K. A. 2003, ApJ, 588, 871

Drake, J. J., Marshall, H. L., Dreizler, S., et al. 2002, ApJ, 572, 996

Dupuis, J., Vennes, S., Bowyer, S., Pradhan, A. K., \& Thej1l, P. 1995, ApJ, 455, 574

Dupuis, J., Vennes, S., Chayer, P., Hurwitz, M., \& Bowyer, S. 1998, ApJ, 500, L45

Finley, D. S., Koester, D., \& Basri, G. 1997, ApJ, 488, 375

Frisch, P. C., Dorschner, J. M., Geiss, J., et al. 1999, ApJ, 525, 492

Gatewood, G. D., \& Gatewood, C. V. 1978, ApJ, 225, 191

Gloeckler, G., \& Geiss, J. 2004, Adv. Space Res., 34, 53

Good, S. A., Barstow, M. A., Holberg, J. B., et al. 2004, MNRAS, 355, 1031

Hébrard, G., Mallouris, C., Ferlet, R., et al. 1999, A\&A, 350, 643
Holberg, J. B., Barstow, M. A., Bruhweiler, F. C., Cruise, A. M., \& Penny, A. J. 1998, ApJ, 497, 935

Holweger, H. 2001, Joint SOHO/ACE workshop Solar and Galactic Composition, AIP Conf. Proc., 598, 23

Hummer, D. G., \& Mihalas, D. 1988, ApJ, 331, 794

Jordan, S., Wolff, B., Koester, D., \& Napiwotzki, R. 1994, A\&A, 290, 834

Kargaltsev, O. Y., Pavlov, G. G., Zavlin, V. E., \& Romani, R. W. 2005, ApJ, 625, 307

Karzas, W. J., \& Latter, R. 1961, ApJS, 6, 167

Lemke, M. 1997, A\&AS, 122, 285

Lodders, K. 2003, ApJ, 591, 1220

Madej, J. 1998, A\&A, 340, 617

Massa, D., \& Fitzpatrick, E. L. 2000, ApJS, 126, 517

Mathis, J. S., Rumpl, W., \& Nordsieck, K. H. 1977, ApJ, 217, 425

Mihalas, D. 1967, ApJ, 149, 169

Napiwotzki, R., Barstow, M. A., Fleming, T., et al. 1993, A\&A, 278, 478

Napiwotzki, R. 1997, A\&A, 322, 256

O’Dwyer, I. J., Chu, Y.-H., Gruendl, R. A., Guerrero, M. A., \& Webbink, R. F. 2003, AJ, 125, 2239

Pease, D. O., \& Drake, J. J. 2003, Chandra-LETGS home page, http: //cxc.harvard.edu/cal/Letg/Hrc gain/

Pease, D. O., Drake, J. J., Johnson, C. O., et al. 2000, Proc. SPIE, 4012, 700

Pease, D. O., Drake, J. J., Kashyap, V. L., et al. 2003, Proc. SPIE, 4851, 157 Pfeffermann, E., Briel, U. G., Hippman, H., et al. 1987, Proc. SPIE, 733, 519

Pons, J. A., Walter, F. M., Lattimer, J. M., et al. 2002, ApJ, 564, 981

Rauch, T., \& Deetjen, J. L. 2003, in Workshop on Stellar Atmosphere Modeling, ed. I. Hubeny, D. Mihalas, \& K. Werner, ASP Conf. Ser., 288, 103

Prieto, M. A., Hasinger, G., \& Snowden, S. L. 1996, A\&AS, 120, 187

Reid, I. N. 1996, AJ, 111, 2000

Reynolds, R. J. 2004, Adv. Space Res., 34, 27

Schuh, S. L., Dreizler, S., \& Wolff, B. 2002, A\&A, 382, 164

Sing, D., Holberg, J. B., \& Dupuis, J. 2002, Continuing the Challenge of EUV Astronomy: Current Analysis and Prospects for the Future, ASP Conf. Ser., 264, 57

Slavin, J. D., \& Frisch, P. C. 2002, ApJ, 565, 364

Snowden, S. L., Freyberg, M. J., Plucinsky, P. P., et al. 1995, ApJ, 454, 643

Snowden, S. L., Turner, T. J., \& Freyberg, M. J. 2001, ApJS, 132, 107

Sofia, U. J., Lauroesch, J. T., Meyer, D. M., \& Cartledge, S. I. B. 2004, ApJ, 605, 272

Suleimanov, V., Madej, J., Drake, J. J., Rauch, T., \& Werner, K. 2006, A\&A, 455,679

Sutherland, R. S. 1998, MNRAS, 300, 321

Trümper, J. 1982, Adv. Space Res., 2, 241

Unsöld, A. 1968, Physik der Sternatmosphären (Berlin: Springer)

van Altena, W. F., Lee, J. T., \& Hoffleit, E. D. 2001, VizieR Online Data Catalog, $\mathrm{I} / 238 \mathrm{~A}$

van Kerkwijk, M. H., \& Kulkarni, S. R. 2001, A\&A, 378, 986

Vennes, S., \& Dupuis, J. 2002, The High Energy Universe at Sharp Focus: Chandra Science, ASP Conf. Ser., 262, 57

Verner, D. A., \& Yakovlev, D.G. 1995, A\&AS, 108, 287

Werner, K., Dreizler, S., Deetjen, J. L., et al. 2003, in Workshop on Stellar Atmosphere Modeling, ed. I. Hubeny, D. Mihalas, \& K. Werner, The ASP Conf. Ser. (San Francisco: ASP), 288, 31

Wilms, J., Allen, A., \& McCray, R. 2000, ApJ, 542, 914

Wolff, B., Jordan, S., Bade, N., \& Reimers, D. 1995, A\&A, 294, 183

Wolff, B., Jordan, S., \& Koester, D. 1996, A\&A, 307, 149

Wolff, B., Koester, D., \& Lallement, R. 1999, A\&A, 346, 969

Wood, M. A. 1995, White Dwarfs, LNP, 443, 41

Yan, M., Sadeghpour, H. R., \& Dalgarno, A. 1998, ApJ, 496, 1044

Zubko, V., Dwek, E., \& Arendt, R. G. 2004, ApJS, 152, 211 